Nach der Säkularisierungsthese. Religion als Herausforderung für schulische Bildung

Après la thèse de la sécularisation : la religion en tant que défi pour l'éducation scolaire

After the Secularization Thesis: Religion as a Challenge for School Education

\title{
Wolfgang Sander
}

\section{Q OpenEdition}

\section{Journals}

\section{Édition électronique}

URL : http://journals.openedition.org/ceg/2835

DOI : $10.4000 /$ ceg. 2835

ISSN : 2605-8359

\section{Éditeur}

Presses Universitaires de Provence

\section{Édition imprimée}

Date de publication : 26 avril 2018

Pagination : 73-88

ISBN : 979-10-320-0160-8

ISSN : 0751-4239

Référence électronique

Wolfgang Sander, « Nach der Säkularisierungsthese. Religion als Herausforderung für schulische Bildung », Cahiers d'Études Germaniques [Online], 74 | 2018, Online erschienen am: 26 April 2018, abgerufen am 25 November 2020. URL : http://journals.openedition.org/ceg/2835 ; DOI : https:// doi.org/10.4000/ceg.2835 


\title{
Nach der Säkularisierungsthese Religion als Herausforderung für schulische Bildung
}

\author{
Wolfgang Sander \\ Universität Gießen
}

In einem deutschen Erfahrungsbericht aus der Islamismusprävention mit muslimischen Jugendlichen werden Schwierigkeiten beim Umgang mit dem Thema Religion in der Schule mit der folgenden Szenerie geschildert: Es zeige

\begin{abstract}
sich in der Schule, dass viele muslimische Schülerinnen und Schüler schwer verstehen können, dass ihre Lehrer oft nicht religiös sind: ,Unsere Lehrer glauben nicht an Gott!', heißt es dann geradezu schockiert. Bei den Pädagogen ist es umgekehrt, wenn sie mitunter genauso fassungslos feststellen: ,Meine Schüler glauben an Gott!'
\end{abstract}

In eine ähnliche Richtung geht eine Beobachtung des Hamburger Lehrerbildners Kurt Edler. Er sieht für die produktive pädagogische Bewältigung von religiösen Konflikten in der Schule einen, wie er schreibt, „schwer erkennbaren Stolperstein. Es ist die Unbeholfenheit der pädagogischen Fachkräfte, die durch die eigene Religionsferne bewirkt wird“. ${ }^{2}$

\section{Die Wiederkehr der Religion in der westeuropäischen Öffentlichkeit}

In solchen Beobachtungen aus der Mikrowelt der Schulen spiegeln sich die beträchtlichen religiös-politischen Verwerfungen, in die die westeuropäischen Gesellschaften in Folge der Einwanderung muslimischer Migranten sowie der globalen Konfrontation mit dem Islamismus geraten sind. Die Migration aus islamisch geprägten Ländern, insbesondere der Türkei, ist lange Zeit vielleicht als ein soziales und kulturelles, nicht aber als ein religiöses Problemfeld wahrgenommen worden. Dafür dürfte die in den 1960er- und 1970er-Jahren

1. Jochen Müller, Götz Nordbruch, Deniz Ünlü, „,Wie oft betest du?' Erfahrungen aus der Islamismusprävention mit Jugendlichen und Multiplikatoren“, in Wael El-Gayar, Karin Strunk (Hrsg.), Integration versus Salafismus. Identitätsfindung muslimischer Jugendlicher in Deutschland, Schwalbach im Taunus, Wochenschauverlag, 2014, S. 148.

2. Kurt Edler, Islamismus als pädagogische Herausforderung, Stuttgart, Verlag W. Kohlhammer, 2015, S. 47. 
in der Öffentlichkeit noch völlig dominant gewesene Säkularisierungsthese verantwortlich gewesen sein. Nach ihr führen in der Moderne der Fortschritt der Wissenschaften auf der einen und der wachsende Wohlstand auf der anderen Seite zu einem zunehmenden Bedeutungsverlust der Religion. Religion erschien in diesem Verständnis als ein vormodernes Phänomen, das durch Aufklärung und Wissenschaft auf lange Sicht zum Verschwinden verdammt sein würde. Mit einem solchen Weltverständnis lohnte eine Beschäftigung mit der Religion der Einwanderer nicht, denn es schien ja als ausgemacht, dass mit der Integration dieser Einwanderer in die säkulare Gesellschaft deren Religiosität mehr und mehr zurückgehen würde.

Davon kann aber auch nach einem halben Jahrhundert nicht die Rede sein. Noch immer zeigen die einschlägigen Studien ein wesentlicher höheres $\mathrm{Ma} \beta$ an Religiosität unter Muslimen in Deutschland und Europa als in der nichtmuslimischen Mehrheit und auch einen deutlich höheren Stellenwert dieser Religiosität für das persönliche Leben. So unterscheiden sich die Jugendkulturen junger Muslime in Deutschland von denen nichtmuslimischer Jugendlicher neben einem traditionelleren Familienbild in erster Linie im Stellenwert der Religion für das eigene Selbstverständnis. ${ }^{3}$ In islamischen Jugendszenen wird vielfach Religiosität öffentlich, kollektiv und selbstbewusst inszeniert, bis hin zum Phänomen des Pop-Islam, der konservative Religiosität mit modernem Lebensstil verbindet. ${ }^{4}$

Vom globalen Konflikt mit dem Islamismus, insbesondere in seinen dschihadistischen Varianten, hat die europäische Öffentlichkeit im Grunde erst mit den Anschlägen vom 11. September 2001 Kenntnis genommen. Auch danach hat mancher die Ereignisse lieber als kriminelle Taten einiger Verirrter abgetan, statt sich mit der offenkundig gewordenen politischen Relevanz von Religion auseinanderzusetzen. Friedrich Wilhelm Graf, von dem die inzwischen fast schon zum geflügelten Wort gewordene Formulierung von der „Wiederkehr der Götter“ ${ }^{5}$ stammt, hat diese geistige Verfassung der intellektuellen Öffentlichkeit etwas bissig so kommentiert:

Der liberale Durchschnittsdenker verstand die Moderne als eine radikal laizistische Welt, eine Welt konsequenter Säkularisierung, in der es Religion höchstens noch in einigen Nischen als Auslaufmodell für Sinnsucher gebe. Doch konnte dieser erfahrungsresistente Irrglaube nur noch in den abgeschotteten Lebenswelten westlicher Intellektueller gedeihen. ${ }^{6}$

Inzwischen hat die Säkularisierungsthese in den Sozial- und Religionswissenschaften drastisch an Bedeutung verloren. Dazu hat auch der

3. Vgl. Hans-Jürgen von Wensierski, „Hip-Hop, Kopftuch und Familie - Jugendkulturen junger Muslime in Deutschland“, in El-Gayar, Strunk, Integration versus Salafismus, S. 38-48.

4. Vgl. Julia Gerlach, Zwischen Pop und Dschihad: Muslimische Jugendliche in Deutschland, Bonn, Bundeszentrale für politische Bildung, 2006.

5. Vgl. Friedrich Wilhelm Graf, Die Wiederkehr der Götter. Religion in der modernen Kultur, München, Beck, 2004.

6. Friedrich Wilhelm Graf, Götter global. Wie die Welt zum Supermarkt der Religionen wird. München, Beck, 2014, S. 238. 
globalisierte Blick in den Wissenschaften beigetragen. In globaler Perspektive betrachtet, kann nämlich von einem Bedeutungsverlust der Religion überhaupt keine Rede sein. Ganz im Gegenteil beobachten wir eine zunehmende Vitalität der Religion in den meisten Teilen der Welt, insbesondere in Afrika, Asien und Lateinamerika. Dies gilt keineswegs alleine oder auch nur in erster Linie für den Islam. Weltweit gibt es ein rasantes Wachstum christlicher Kirchen und Bewegungen. Es betrifft zu erheblichen Teilen den freikirchlichen Protestantismus, aber auch die Aufmerksamkeit und der Zustrom, den jede öffentliche Messe des Papstes weltweit hervorruft, strafen die Säkularisierungsthese Lügen.

Die große und erklärungsbedürftige Ausnahme ist Europa, vor allem Westund Nordeuropa. Hier gab es in der gesellschaftlichen Entwicklung der letzten zwei Jahrhunderte Anhaltspunkte, die dem von Graf aufgespießten Irrglauben westlicher Intellektueller Nahrung boten, ja ihn zu einem kulturell nahezu hegemonialen Konzept werden ließen. Mit Blick darauf gilt es zunächst genauer hinzusehen, nach Gründen und nach begrifflichen Alternativen zu suchen, wenn man heute nach Religion als Herausforderung fragt.

\section{Zwischen Säkularismus und Fundamentalismus: das religiös-säkulare Spannungsfeld}

Wenn man sich mit Religion und Säkularität befasst, stößt man schnell auf sehr unterschiedliche Verständnisse und Verwendung der bei diesem Themenfeld zentralen Begriffe Religion, Glaube und Säkularität. Es soll deshalb zunächst skizziert werden, wie und in Anlehnung an wen diese zentralen Begriffe nachfolgend verwendet werden.

Religionen sind in der Menschheitsgeschichte ubiquitär. Gesellschaften ohne Religion hat es, soweit wir wissen, nie gegeben. Wie groß man Einfluss und Verbreitung der Religion jeweils einschätzt, hängt freilich von deren näherer begrifflichen Bestimmung hat. Die Erklärung „Nostra Aetate - Über das Verhältnis der Kirche zu den nichtchristlichen Religionen“ des Zweiten Vatikanischen Konzils definiert Religion von den Fragen her, auf die sie Antworten gibt:

\footnotetext{
Was ist der Mensch? Was ist Sinn und Ziel unseres Lebens? Was ist das Gute, was die Sünde? Woher kommt das Leid, und welchen Sinn hat es? Was ist der Weg zum wahren Glück? Was ist der Tod, das Gericht und die Vergeltung nach dem Tode? Und schließlich: Was ist jenes letzte und unsagbare Geheimnis unserer Existenz, aus dem wir kommen und wohin wir gehen? ${ }^{7}$
}

Diese Fragen stellen sich aufgrund unserer Endlichkeit, also eben weil wir kommen und gehen. Fasst man den Religionsbegriff weit, dann schließt er alle Antworten auf die Frage nach Sinn angesichts der Endlichkeit unseres Lebens ein. In einem solchen Verständnis definiert etwa Volker Ladenthin „Religion

7. Vgl. [http://www.vatican.va/archive/hist_councils/ii_vatican_council/documents/vat-ii_ decl_19651028_nostra-aetate_ge.html]. 
als die Frage nach dem Umgang mit unserer Endlichkeit und nach Sinn“" ${ }^{\text {. Aus }}$ diesem weiten Religionsbegriff folgt, dass Menschen nicht nicht-religiös sein können, weil sich alle Menschen auf die eine oder andere Weise mit dieser Frage auseinandersetzen müssen. Für Ladenthin sind denn auch alle Menschen religiös. Allerdings gehören sie deshalb nicht zwingend einer organisierten Religion an. Solche organisierten, institutionalisierten Religionen nennt Ladenthin ,Konfessionen: Konfessionen sind für ihn Glaubensgemeinschaften; ,Glaube wäre hiernach der engere Begriff als ,Religion:

Man kann es freilich auch gerade umgekehrt sehen und mir scheint diese Umkehrung weiterführender $\mathrm{zu}$ sein. So schreibt etwa der islamische Religionswissenschaftler Reza Aslan:

Religion ist etwas anderes als Glaube. Religion ist die erzählte Geschichte des Glaubens. Ein institutionalisiertes Gefüge aus Symbolen und Metaphern (Ritualen und Mythen) in einer Sprache, die es einer Glaubensgemeinschaft ermöglicht, einander ihre Begegnung mit dem Numinosen, dem Göttlichen, mitzuteilen. ${ }^{9}$

Hiernach beziehen sich Religionen nicht auf jede Art von Sinnerleben, sondern auf eine transzendente und absolute Realität, die die Vergänglichkeit der erfahrbaren Wirklichkeit und die Endlichkeit des menschlichen Lebens überschreitet. Da diese transzendente Realität weder empirisch noch begrifflich-logisch direkt zu erkennen ist, vermitteln Religionen Glaubenserfahrungen mittels Erzählungen, Symbolen, Bildern und Ritualen.

Der Vorteil eines solchen engeren Verständnisses von Religion ist es, dass es eine klarere Abgrenzung von Religion und Nicht-Religion erlaubt. Allerdings erkauft man sich damit das Problem, wie Glaubenssysteme begrifflich gefasst werden können, die sich nicht als religiös verstehen, aber funktional äquivalent zu Religionen sind, weil sie Sinnversprechen anbieten und dabei oftmals auch Symbolsysteme und Kommunikationsmuster der Religionen imitieren. Dies ist für das Verständnis der religiösen Situation in so genannten säkularen Gesellschaften von einiger Relevanz. Mir erscheint es als sinnvoll, bei solchen Glaubenssystemen im Anschluss an Paul Tillich von „Quasi-Religionen“10 zu sprechen.

Nach einem solchen Begriffsverständnis können Menschen durchaus ohne Religion leben, prinzipiell wohl auch ohne Quasi-Religion. Können sie aber auch ohne Glauben leben? Wohl eher nicht. Der Philosoph Volker Gerhardt hat kürzlich überzeugend gezeigt, dass ohne Glauben für Menschen Handeln in der Welt kaum möglich ist. Gerhardts These ist, „dass jede und jeder irgendetwas immer glaubt, sobald er ernsthaft etwas tut oder lässt" ${ }^{11}$. Zwar benötigen wir für

8. Volker Ladenthin, Wozu religiöse Bildung heute? Sieben Versuche, an der Endlichkeit zu zweifeln, Würzburg, Echter, 2014, S. 151.

9. Reza Aslan, Kein Gott außer Gott. Der Glaube der Muslime von Muhammad bis zur Gegenwart, München, Beck, 2006, S. 18.

10. Vgl. die knappe, treffende Defınition unter [http://universal_lexikon.deacademic.com/289215/ Quasireligion] (Stand: 25. Juni 2016).

11. Volker Gerhardt, Der Sinn des Sinns. Versuch über das Göttliche, München, Beck, 2014, S. 9. 
Handeln Wissen; aber wenn es ernsthaft um etwas geht bei dem, was wir tun oder lassen wollen, müssen unsere Gründe den Bereich des Wissens überschreiten und von Vertrauen auf etwas getragen sein. Wir mögen wissen - oder zu wissen glauben, hier beginnen schon die Schwierigkeiten -, dass wir jemanden lieben, aber die Entscheidung dafür oder dagegen, sich auf eine verbindliche Beziehung einzulassen, basiert auf dem Glauben an unsere Vorstellungen von unserer Zukunft. Nicht anders verhält es sich, um weitere Beispiele zu nennen, mit der Entscheidung für oder gegen Kinder, dem Anstreben eines beruflichen Weges oder mit einer politischen Handlung. Immer benötigen wir Wissen, um handeln zu können; und immer ist dieses Wissen systematisch unvollständig und wir wären handlungsunfähig, wollten wir erst dann entscheiden, wenn wir über vollständiges Wissen zu einer Situation verfügen. Wir gründen unser Handeln letztlich auf unsere Vorstellungen von der Welt; und diese Vorstellungen enthalten Elemente, denen wir vertrauen müssen, weil wir die Welt als Ganzes gar nicht kennen können.

Die Sache wird nicht einfacher dadurch, dass jedes neue Wissen immer auch neues Nicht-Wissen mit sich bringt. Deshalb betont Gerhardt den Zusammenhang zwischen Glauben und Wissen: „Kein Wissen kommt ohne Glauben aus, und es ist kein Glauben denkbar, der nicht auf Wissen beruht." 12 Nicht notwendigerweise muss es sich dabei um einen religiösen Glauben handeln. Aber Volker Gerhardt spricht schon von der Erfahrung des Göttlichen, wenn die Welt „uns in einer exemplarischen Ansicht als übergroß und übermächtig, vielleicht sogar als staunenswert, schön und erhaben gegenübertritt" ${ }^{13}$.

Es greift offenbar zu kurz, wenn man trennscharf zwischen Religion und Glauben auf der einen und Säkularität auf der anderen Seite unterscheiden wollte. Die Verteilung religiöser und säkularer Bereiche in einer Gesellschaft ist kein Nullsummenspiel, bei dem die Ausweitung des einen automatisch zu Lasten des anderen gehen muss. Eben dies aber scheint mir eine Kernannahme der Säkularisierungsthese gewesen zu sein: säkulares Wissen aus den Wissenschaften werde notwendigerweise religiöses Weltverstehen verdrängen. Aber so einfach lagen und liegen die Dinge nicht, auch nicht in der Geschichte der neuzeitlichen Wissenschaften und der Aufklärung in Europa. So konnten in der Epoche der Aufklärung deren prominente Vertreter mit der Vorstellung, das Zeitalter der Vernunft werde den Glauben an Gott zum Verschwinden bringen, nichts anfangen. Voltaire sah den Atheismus auf einer Stufe mit Aberglauben und Fanatismus ${ }^{14}$ und Robespierre teilte nach der Französischen Revolution zwar deren antiklerikalen Affekt, aber als er auf der Basis der Vernunft einen neuen Kult einführen wollte, ließ er eine symbolische Darstellung des Atheismus verbrennen ${ }^{15}$. Immanuel Kant, der wie kein anderer Philosoph der Aufklärung

12. Ibid., S. 29.

13. Ibid., S. 21.

14. Vgl. Karen Armstrong, Die Geschichte von Gott. 4000 Jahre Judentum, Christentum und Islam, München, Pattloch, 2012, S. 465.

15. Vgl. Jörg Lauster, Die Verzauberung der Welt. Eine Kulturgeschichte des Christentums, München, Beck, 2014, S. 453. 
Vernunft und kritisches Denken ins Recht setzen wollte, betonte zugleich, er habe das Wissen aufheben müssen, um dem Glauben Platz zu machen. ${ }^{16}$ Auch die frühen Astronomen, die das moderne naturwissenschaftliche Weltbild mitbegründeten, Kopernikus, Kepler und auch Galileo Galilei, verstanden ihre Arbeiten keineswegs in einem antireligiösen Sinn.

Allerdings waren die Reaktionen der Kirchen auf die Aufklärung und die neuzeitlichen Wissenschaften bis ins 20. Jahrhundert hinein vielfach mehr als unglücklich, um das mindeste zu sagen. Die Neigung der Kirchen, auf die neuen Herausforderungen mit Abwehr, Pflege einer konservativen Volksfrömmigkeit und wörtlicher Schriftgläubigkeit zu reagieren, beförderte eine religiöse Einstellung, für die im 20. Jahrhundert der Begriff des Fundamentalismus aufkam. Jahrzehnte bevor er auf antimoderne Bewegungen im Islam bezogen wurde, entstand er als Selbstbezeichnung rigoristisch bibelgläubiger Christen in den USA. Die katholische Kirche wiederum führte durch das ganze 19. Jahrhundert hindurch einen letztlich vergeblichen Abwehr- und Kulturkampf gegen den Geist der Moderne, dessen Höhepunkt die Verkündigung der Dogmen von der Unbefleckten Empfängnis Mariens im Jahr 1854 und der Unfehlbarkeit des Papstes im Jahr 1870 waren. Aber schon diese beiden Jahreszahlen - 1854 und 1870 - weisen darauf hin, dass die Konfliktkonstellation zwischen fundamentalistischen religiösen Glaubenshaltungen auf der einen Seite und einem säkularen Weltverständnis, das sich anti-religiös positioniert, ihre Wurzeln im Europa des 19. Jahrhunderts hat. Weder ist Wissenschaft per se anti-religiös, noch ist Religion per se vernunftsund wissenschaftskritisch oder gar fundamentalistisch.

Dies lässt sich gerade an der christlich-jüdischen Tradition in Europa sehr gut zeigen. Die Frankfurter Rabbinerin Elisa Klapheck weist auf das „religiössäkulare Spannungsverhältnis des Judentums“"17 hin. Sie sieht die Spannung schon sehr früh in zwei Polen angelegt: in Gottes monotheistischem Anspruch einerseits und dem freien Willen des Menschen andererseits. Weiterhin enthält die Thora zwar Gottes Offenbarung; deren Auslegung aber wird im Talmud unter Generationen von Rabbinern in einem rein säkularen System von Argumentation und Mehrheitsentscheidung für die jeweilige Zeit erörtert. Schließlich impliziert der Monotheismus selbst von Beginn an ein säkulares Verständnis der Welt: Weil sie Geschöpf des einen Gottes ist, gibt es in ihr keine Götter mehr. Eben dies ist der theologische Kern der ersten Schöpfungsgeschichte in der Bibel: Die ganze Natur, Sonne und Sterne, Pflanzen, Tiere und Menschen, sind Geschöpfe Gottes und eben deshalb selbst keine anbetungswürdigen Götter.

Auch das Christentum bekräftigt von Beginn an das Eigenrecht des Säkularen. Das Jesuswort „Gebt dem Kaiser, was des Kaisers ist, und Gott, was Gottes ist“ (Mk. 12,17) markiert dieses Eigenrecht. Es verweist aber auch auf die religiössäkulare Spannung, denn es muss immer wieder neu geklärt werden, was genau

16. In der Kritik der reinen Vernunft; vgl. zu Kant in diesem Zusammenhang ausführlicher Gerhardt, Der Sinn des Sinns, S. 167 ff.

17. Elisa Klapheck, „Das religiös-säkulare Spannungsverhältnis des Judentums“, in dies., Ruth Calderon, Säkulares Judentum aus religiöser Quelle, Berlin, Hentrich und Hentrich, 2015, S. 9-47. 
des Kaisers und was Gottes ist. Tatsächlich ist dies ein immer wiederkehrendes Thema in der europäischen Geschichte gewesen. Einer der zentralen Konflikte der mittelalterlichen Geschichte, der Investiturstreit zwischen Kaiser und Papst im 11. und 12. Jahrhundert, wäre gar nicht möglich gewesen ohne die Unterscheidung zwischen einer weltlichen und einer geistlichen Macht. 400 Jahre später wird Martin Luther zwischen dem Reich Gottes und dem Reich der Welt unterscheiden, in denen der Christ gleichzeitig lebt, ein Thema, das die protestantische Theologie bis in unsere Zeit bewegt.

Offenbar ist das Problem, wie sich religiöse und säkulare Bereiche der Gesellschaft zueinander verhalten sollen, in der europäischen Geschichte erheblich älter als die Säkularisierungsthese. Aber ein weiterer, wesentlicher Aspekt kommt noch hinzu. Er betrifft die Frage, was an den säkularen Vorstellungen in der Moderne, die sich in den Konflikten der letzten Jahrhunderte gegen das etablierte Christentum wandten, selbst christliche Wurzeln hat. Der britische Ideengeschichtler Larry Siedentop hat kürzlich in einer luziden Studie nachgezeichnet, wie tief die der Aufklärung und dem modernen Liberalismus so wichtigen Ideen im frühen Christentum wurzeln, insbesondere in der Theologie von Paulus - Ideen wie das Konzept des Individuums, die Gleichheit der Menschen, Gewissensfreiheit und der Universalismus moralischer Normen. ${ }^{18}$ Die Beziehung Gottes zu den Menschen wird erst mit dem Christentum als eine zwischen Gott und jedem einzelnen Menschen gedacht, unabhängig von dessen sozialem Status. Ohne diese „moralische Revolution“, so Siedentop, mit der sich das frühe Christentum radikal von der nach Familien und anderen Kollektiven geordneten Glaubenswelt der Antike unterschied, wäre seine gewaltfreie Durchsetzung im Römischen Reich kaum möglich gewesen.

Gewiss hat die nachfolgende Geschichte des Christentums auch andere, dunklere Seiten. Es geht hier nicht um eine nostalgische Idealisierung der komplexen, vielschichtigen und spannungsreichen Realgeschichte des Christentums. Aber es gibt gute Gründe für die These, dass die säkularen Bewegungen der Neuzeit in Europa, so Siedentop, „die christlichen Moralvorstellungen gegen eine privilegierte, auf Zwang basierende Stellung der Kirchen“" ${ }^{19}$ geltend machten. Ein gutes Beispiel hierfür ist die Französische Revolution. Ihr Aufstand gegen das Ancien Regime richtete sich auch gegen die mit diesem Regime verwobene katholische Kirche. Aber ihre Parole „Freiheit, Gleichheit, Brüderlichkeit“ kann ihre Herkunft aus dem christlichen Menschenbild nicht verleugnen. Dennoch kam es ab 1792 in Frankreich zu einer massiven und brutalen Christenverfolgung, die erst 1799 unter Napoleon beendet wurde.

So hat die säkulare Welt des heutigen Europas zwar selbst religiöse Wurzeln. Aber zugleich entwickeln sich in der Neuzeit Formen säkularen Denkens, die mit

18. Vgl. Larry Siedentop, Die Erfindung des Individuums. Der Liberalismus und die westliche Welt, Stuttgart, Klett-Cotta, 2015.

19. Ibid., S. 449. Siedentop spricht in diesem Zusammenhang vom „Säkularismus“. Aus den nachfolgend genannten Gründen kann ich dieser begrifflichen Zuschreibung nicht uneingeschränkt folgen. 
einem starken antireligiösen Affekt verbunden sind. Es scheint mir deshalb sinnvoll zu sein, zwischen Säkularität und Säkularismus zu unterscheiden. ${ }^{20}$ Säkular heißt bekanntlich schlicht ,weltlich' Säkulare gesellschaftliche Bereiche und Institutionen sind solche, die in der weltlichen Verantwortung des Menschen liegen. Sie können durchaus in normativer Hinsicht religiöse Wurzeln haben, folgen aber in ihrer Praxis einer nicht-religiösen Eigenlogik. Gleichwohl können in ihnen Menschen leben und arbeiten, die religiös geprägt sind. Der Säkularismus hingegen ist eine religionsfeindliche Weltanschauung. Er gründet sich auf die Glaubenshaltung eines metaphysischen Naturalismus ${ }^{21}$, erhebt einen umfassenden Erklärungs- und Gestaltungsanspruch für Natur und Gesellschaft und will deshalb die Religion aktiv aus dem öffentlichen Leben verdrängen. Teilweise nimmt er selbst Züge einer Quasi-Religion an. Dann wird die Evolution als quasi-göttlicher Akteur verstanden, der sich in den Naturwissenschaften offenbart; das Erlösungsversprechen eines solchen quasi-religiösen Säkularismus ist die immer weitere Verringerung und schlussendliche Beseitigung des menschlichen Leidens in der vollendeten technisch-wissenschaftlichen Zivilisation. Der Säkularismus ist das säkulare Gegenstück zum religiösen Fundamentalismus. Beide lassen sich als Pathologien der Moderne verstehen.

In der Moderne wurde das religiös-säkulare Spannungsfeld neu geordnet. Neue säkulare Institutionen wie der Rechtsstaat, die modernen Sozialsysteme und die freie Wissenschaft entstanden, Institutionen, die nur politische oder religiöse Extremisten missen möchten. Die großen Volkskirchen in Europa wurden geschwächt, aber zugleich vervielfältigte sich das Christentum. Dabei entstanden auch neue säkular-religiöse Verbindungen wie beispielsweise der Kulturprotestantismus oder die Verarbeitung religiöser Erfahrungen in einer äußerlich säkularen Kunst. Aber dabei blieb es nicht. Die Schwächung der großen Kirchen öffnete auch einen Raum für eine Vielzahl obskurer Quasi-Religionen. Es ist die große Illusion des Säkularismus, dass dort, wo Religion verdrängt wird, die Vernunft Platz greife. Der Wille zu glauben, hat sich oft ganz andere Wege gebahnt. Manche davon haben sich als katastrophal erwiesen, wie der Nationalsozialismus und der Stalinismus, deren quasi-religiöser Charakter schon oft beschrieben wurde. Andere sind bislang weniger gefährlich, tragen aber durchaus ein Gefahrenpotenzial in sich, wie einige der Neo-Mythen, die Linus Hauser analysiert hat. ${ }^{22}$ Wieder andere mögen als harmlose Spinnereien gelten, wie die vielen Formen des Esoterischen und des schieren Aberglaubens, die so manches Regal in den Buchhandlungen füllen, oder die privaten Bastelreligionen, die willkürlich Elemente aus verschiedenen religiösen Traditionen zu

20. Vgl. dazu bereits Friedrich Gogarten, Verhängnis und Hoffnung der Neuzeit, Stuttgart, Vorwerk, 1958.

21. Vgl. zur Analyse und Kritik des metaphysischen Naturalismus Thomas Nagel, Geist und Kosmos. Warum die materialistische neodarwinistische Konzeption der Natur so gut wie sicher falsch ist, Berlin, Suhrkamp, 2013.

22. Vgl. Linus Hauser, Kritik der neomythischen Vernunft, 3 Bde., Paderborn, Schöningh, 2005 (2. Aufl.), 2009 und 2016. 
unausgegorenen Glaubenssystemen zusammenrühren, oder die Sakralisierung von Pop-Stars oder Fußballvereinen.

Und dann ist da noch jener Gott, auf dessen Popularität schon Martin Luther hingewiesen hat und den er mit dem Wort ,Mammon' bezeichnete: Geld und Gut, Leistung und Erfolg. Denn, so Luther weitsichtig mit dem vielzitierten Satz aus seinem Großen Katechismus: „Woran du nun dein Herz hängest und worauf du dich verlässest, das ist eigentlich dein Gott." Dies war nicht moralisch, sondern durchaus analytisch zu verstehen. Dass dies auch heute eine fruchtbare analytische Perspektive ist, zeigt eine Arbeit des Wirtschaftswissenschaftlers Oliver Fohrmann, die sich unter religionstheoretischen Aspekten mit der Vergöttlichung des Geldes beschäftigt. ${ }^{23}$

Auf diese Gemengelage in den europäischen Gesellschaften treffen nun in den letzten Jahrzehnten hochreligiöse Migrationskulturen. Sie mischen das religiös-säkulare Spannungsfeld neu auf und erweitern es um die Dimension religiöser Vielfalt mit Blick auf die großen Weltreligionen. Es liegt auf der Hand, dass dies die Schule unter dem Aspekt der religiösen Bildung vor grundlegende Herausforderungen stellt.

\section{Bildung und Religion - religiöse Bildung im religiös-säkularen Spannungsfeld}

Fragt man nach dem Zusammenhang von Bildung und Religion, so ist eine erste Antwort: Die Idee der Bildung, so wie sie seit dem Neuhumanismus die pädagogische Diskussion beschäftigt, hat in Europa einen religiösen Ursprung. Sie erscheint im Hochmittelalter in der christlichen Mystik bei Meister Eckart. Er greift auf die Imago-dei-Lehre zurück, nach der der Mensch als Ebenbild Gottes geschaffen ist. Jeder Mensch trägt das Bild Gottes in seiner Seele, aber er muss es erst finden, indem er über verschiedene Stufen seine Erkenntnisfähigkeit entwickelt. Modern gesprochen: Jeder Mensch ist bildsam, aber er muss sich erst noch bilden. Bei Meister Eckart verschmilzt in diesem Prozess die Seele mit Gott, dabei bildet sich der Mensch in Gott ein. Dieser Weg der Bildung ist ein Weg der Selbsterkenntnis, die mit der Erkenntnis Gottes identisch ist. Man könnte auch sagen: Durch Bildung verknüpft sich der Einzelne mit dem Ganzen der Welt, das in Gott ist.

Die neuhumanistische Vorstellung von Bildung, wie sie von Wilhelm von Humboldt klassisch formuliert wurde, lässt sich durchaus als eine säkulare Adaption dieser Imago-dei-Lehre deuten:

Die letzte Aufgabe unseres Daseyns: dem Begrif der Menschheit in unsrer Person, sowohl während der Zeit unsres Lebens, als auch noch über dasselbe hinaus, durch die Spuren des lebendigen Wirkens, die wir zurücklassen, einen so grossen Inhalt, als möglich,

23. Vgl. Oliver Fohrmann, Im Spiegel des Geldes. Bildung und Identität in Zeiten der Ökonomisierung, Bielefeld, transcript, 2016. 
zu verschaffen, diese Aufgabe löst sich allein durch die Verknüpfung unsres Ichs mit der Welt zu der allgemeinsten, regesten und freiesten Wechselwirkung. ${ }^{24}$

Auch Humboldt sieht den Bildungsprozess des Individuums als einen der Vervollkommnung; und auch er bezieht diesen Bildungsprozess jedes Einzelnen auf das Ganze der Welt. Jeder Einzelne soll durch Bildung befähigt werden, dem Begriff der Menschheit in seinem Leben „einen so grossen Inhalt, als möglich, zu verschaffen“, er soll also gewissermaßen zu einem Bild der ganzen Menschheit werden. Darin bestehe, so Humboldt, die „letzte Aufgabe unseres Daseyns“. Von Gott ist hier explizit zwar nicht mehr die Rede, aber die theologischen Anklänge und die strukturelle Ähnlichkeit der Argumentation sind doch deutlich zu erkennen.

Solche Säkularisierungen religiöser Vorstellungen prägen auch andere Grundkonzepte, die für das moderne Schulwesen tragend sind. Die Idee einer Unterrichtspflicht für alle findet sich erstmals bei Martin Luther, der sie mit dem protestantischen Konzept des Priestertums aller Gläubigen begründete. Bei Comenius fielen Welt- und Gotteserkenntnis in eins, war Wissenschaft nur der Nachvollzug von Gottes Schöpfungstat; aus dieser Sicht heraus konnte er den im 17. Jahrhundert unerhörten Gedanken formulieren, die Schulen hätten alle Menschen alles zu lehren. Ohne Risiken sind die Säkularisierungen solcher religiös begründeten Motive im modernen pädagogischen Denken freilich nicht. So ist die Pädagogik immer wieder der Versuchung erlegen, an Erziehung und Bildung umfassende Heilserwartungen zu knüpfen, sich also die Beseitigung aller Übel in einer (wie auch immer gearteten) vollkommenen Gesellschaft auf dem Wege der Neuerziehung der nachwachsenden Generation zu erwarten. Pädagogisches Denken kann in solchen Vorstellungswelten durchaus quasireligiöse Züge annehmen: „Erziehung als Erlösung““25.

Diese knappen Hinweise sollen lediglich illustrieren, dass die Idee der Bildung für alle durch Schulen in der europäischen Tradition in das religiössäkulare Spannungsfeld eingelassen ist. Dies sagt noch nicht viel über die Frage, wie Schulen heute mit Religion auf eine bildungsförderliche Weise umgehen können. Aber es macht vielleicht deutlich, dass Bildung und Religion in einer Beziehung miteinander gesehen werden müssen, bei der es um mehr geht als um die Vermittlung von ein wenig oberflächlichem religionskundlichem Wissen.

In Bildungsprozessen verbinden sich Menschen mit der Welt. Sie setzen sich dabei mit der Welt auf Weisen auseinander, die ihre Erfahrungen und ihr Verstehen erweitern und sie in ein reflexives Verhältnis zu dem versetzen, was ihnen an Kultur im weitesten Sinn begegnet. Es wäre widersinnig, wollte die Schule dabei die fundamentalen Fragen ausklammern, auf die Religionen

24. Wilhelm von Humboldt, „Theorie der Bildung des Menschen. Bruchstück“, in Heinz-Elmar Tenorth (Hrsg.), Allgemeine Bildung. Analysen zu ihrer Wirklichkeit, Versuche über ihre Zukunft, Weinheim / München, Juventa, 1984, S. 34.

25. Vgl. beispielsweise die einschlägige kritische Analyse zur Reformpädagogik bei Meike Sophia Baader, Erziehung als Erlösung. Transformationen des Religiösen in der Reformpädagogik, Weinheim / München, Juventa, 2005. 
Antworten suchen; und es wäre angesichts der ubiquitären Verbreitung von Religion in Geschichte und Gegenwart ebenso widersinnig, wollte man eben diese Antworten in Bildungsangeboten ausklammern. Dietrich Benner formuliert es so:

Wenn wir [...] die Auseinandersetzung und Reflexion der eigenen Endlichkeit als Erfordernis einer reflektierenden Bildung ansehen, dann ist die Besinnung auf die Abhängigkeit des Menschen vom Absoluten - in welchen Formen auch immer - ein genuiner Horizont menschlicher Bildung. ${ }^{26}$

Die Hauptfeinde einer solchen reflektierenden religiösen Bildung sind religiöser Fundamentalismus einerseits und Säkularismus andererseits. Beide verbindet die Überzeugung, alles Wichtige schon zu wissen, und die Neigung, das unvermeidliche Nicht-Wissen durch bloße Bekenntnisse zu überspielen; beide verbindet auch Dogmatismus und eine Tendenz zur Intoleranz gegenüber Andersdenkenden und Andersgläubigen. Religiöse Bildung in der Schule hat demgegenüber die Aufgabe, junge Menschen in jeweils geeigneten Ausschnitten mit dem Reichtum religiöser Überlieferungen als Ressourcen für die Entwicklungen des eigenen Weltverstehens bekannt zu machen. Mit Blick auf religiös-politischen Extremismus wird von religiöser Bildung in der Schule ein Beitrag zur Prävention zu erwarten sein. Aktuell betrifft dies in erster Linie den islamistischen Extremismus. ${ }^{27}$

Religiöse Bildung muss vom Faktum religiöser Pluralität ausgehen. Hierbei kann sie einen gewichtigen Beitrag zur Förderung religiöser Toleranz und damit zum friedlichen Zusammenleben in multireligiösen Gesellschaften leisten. Hierfür ist es notwendig, dass Schülerinnen und Schüler lernen, den je eigenen Wahrheitsanspruch in Glaubensfragen mit der Akzeptanz konkurrierender Wahrheitsansprüche zu verbinden. Dies setzt freilich voraus, dass sie sich mit eigenen Wahrheitsansprüchen in Glaubensfragen unter Bezug auf die jeweilige religiöse Tradition überhaupt reflexiv auseinandergesetzt und eine, wenn auch vielleicht vorläufige, Position gefunden haben. Zu glauben, man könne religiösem Fundamentalismus und religiös-politischem Extremismus mit Gleichgültigkeit und religiöser Indifferenz begegnen, wäre jedenfalls eine gefährliche Illusion.

26. Dietrich Benner, Bildung und Religion. Nur einem bildsamen Wesen kann ein Gott sich offenbaren, Paderborn, Schöningh, 2014, $20 \mathrm{f}$.

27. Vgl. zur aktuellen einschlägigen Diskussion in Deutschland den kritischen Literaturbericht bei Wolfgang Sander, „Prävention von islamistischem Extremismus durch Schulkultur und Unterricht in den gesellschaftswissenschaftlichen Fächern - Befunde und Perspektiven“, zeitschrift für didaktik der gesellschaftswissenschaften (zdg) 1, 2016, S. 124-136. 


\section{Perspektiven religiöser Bildung in der Schule}

Für die Repräsentation von Religion im Bildungsangebot der Schule lassen sich idealtypisch vier Varianten unterscheiden.

\section{Verdrängung}

Religion wird nicht als expliziter Gegenstand des schulischen Curriculums verstanden. Schulische Bildung wird als gänzlich unabhängig von religiösen Bezügen, möglicherweise auch in dezidiertem Gegensatz zu religiösen Glaubensvorstellungen konzipiert, unter Umständen sogar als Instrument zur Kritik der Religion gesehen. Schulische Bildung wird hier meist in einem säkularistischen Begründungskontext gedacht; Religion wird dann - wenn überhaupt - allenfalls als Privatangelegenheit der Individuen respektiert. Diese Variante findet sich im 20. Jahrhundert unter gänzlich unterschiedlichen weltanschaulichen Vorzeichen in den dezidiert religionsfeindlichen marxistischleninistischen Systemen in Ostereuropa und im Nationalsozialismus, aber auch im französischen Laizismus ${ }^{28}$ und in der kemalistisch geprägten Türkei von 1924 bis zur sukzessiven Wiedereinführung des Religionsunterrichts unter strikter staatlicher Kontrolle ab Ende der 1940er-Jahre.

\section{Katechese}

Schule wird hier als Ort der Verbreitung und Stabilisierung des im jeweiligen Staatsgebiet vorherrschenden religiösen Glaubens verstanden. Der kirchlich verantwortete oder zumindest maßgeblich beeinflusste Religionsunterricht wird als das hierfür wichtigste Fach angesehen. In Europa war diese Variante bis zur Französischen Revolution durchweg für das Schulwesen prägend. In Deutschland erfuhr sie nach der Erfahrung des Nationalsozialismus in den 1950er-Jahren noch einmal eine Renaissance; besonders prägnant war dies im protestantischen Bereich in der religionspädagogischen Konzeption der ,Evangelischen Unterweisung“ der Fall. Religionsunterricht wurde hier als „Kirche in der Schule“ ${ }^{29}$ gedacht. Dies wurde allerdings von den Vertretern dieser Konzeption nicht als Ausdruck kirchlicher Machtpolitik verstanden. Sie wurde vielmehr zunächst als Reaktion auf den Kulturschock des Ersten Weltkriegs und später zusätzlich auf die Erfahrungen im Widerstand der Bekennenden Kirche gegen die NS-Herrschaft konzipiert. „Kirche in der Schule“ sollte ein Gegenkonzept gegen einen überzogenen bürgerlichen Bildungsidealismus wie auch gegen die Allmacht des Staates in der Erziehung der Jugend sein. In der Rechtsstellung des

28. Vgl. die differenzierte Darstellung hierzu bei Bernd Schröder, „Religion(en) und Schule in Frankreich“, Theo-Web 2, 2015 [http://www.theo-web.de/zeitschrift/ausgabe-2005-02/ schroeder_frkr_relu-schul-1-thk-mr-rh.pdf], [Stand: 12. März 2017].

29. Martin Rang, Handbuch für den biblischen Unterricht, Bd. 1, Tübingen, Furche, 1947 [1939], S. 106. 
Religionsunterrichts in Deutschland wirkt die Variante der Verkündigung insofern nach, als er nach Artikel 7 (2) des Grundgesetzes „ordentliches Lehrfach“ ist, das „in Übereinstimmung mit den Grundsätzen der Religionsgemeinschaften erteilt“ wird. Zugleich allerdings stellt das Grundgesetz in Artikel 7 (1) die Teilnahme am Religionsunterricht in die Entscheidungsmacht der Erziehungsberechtigten. De facto wird er deshalb in aller Regel als konfessioneller, also zunächst einmal als evangelischer oder katholischer Religionsunterricht erteilt, der sich inhaltlich allerdings in den letzten Jahrzehnten vom Ansatz der Verkündung gelöst hat (vgl. unten 4.). Weil es seit den 1970er-Jahren verstärkt zu Abmeldungen kam, für die vermutlich bei weitem nicht immer Gewissensgründe ausschlaggebend waren, wurde für diejenigen Schülerinnen und Schüler, die nicht am Religionsunterricht teilnehmen, in den meisten Bundesländern ein obligatorisches Ersatzfach eingeführt, überwiegend unter der Bezeichnung ,Ethik؛ In jüngster Zeit wird in mehreren Bundesländern zusätzlich mit islamischem Religionsunterricht experimentiert, hier und da gibt es auch jüdischen.

\section{Information}

In dieser Variante soll Religion zwar Gegenstand des schulischen Curriculums sein, sei es als eigenes Fach, sei es als Themenbereich anderer Fächer. Dieser Unterricht soll auf eine ,neutrale“ Weise im Sinne einer ,Religionskunde“ wissenschaftsorientiert Wissen über Religion(en) vermitteln. Er wendet sich damit zugleich an alle Schülerinnen und Schüler unabhängig von deren Religionszugehörigkeit. Vereinzelt gibt es in Deutschland solche Fächer, so in Bremen und Brandenburg, die sich auf eine Ausnahmeklausel im Grundgesetz berufen, nach der abweichende Regelungen zum Religionsunterricht möglich sind, wenn im jeweiligen Bundesland vor 1949 bereits eine andere Regelung bestand als der nach dem Grundgesetz gewollte Unterricht in Übereinstimmung mit den Grundsätzen der jeweiligen Religionsgemeinschaft. Zu dieser Variante gehört auch die informierende Thematisierung von Religionen im Ersatzfach Ethik in anderen Bundesländern.

\section{Dialog}

Diese Variante prägt das Selbstverständnis des heutigen schulischen Religionsunterrichts in Deutschland in starkem Maße. Hiernach ist die Auseinandersetzung mit Religion in diesem Fach in mehrfacher Hinsicht durch dialogische, durchaus spannungsreiche Bezüge zwischen verschiedenen Traditionen, Lebenswelten und Weltverständnissen geprägt: beispielsweise zwischen religiösen Symbolen, Narrationen und Theorien einerseits und Lebenssituationen heutiger Menschen sowie heutiger gesellschaftlicher und politischer Problemlagen andererseits; zwischen eigener Konfessionalität und religiösem Pluralismus; zwischen Religion und Religionskritik; zwischen Glaube und Wissenschaft. In der katholischen Religionspädagogik wird diese 
dialogische Struktur oft auch als „Korrelation“30 bzw. Korrelationsdidaktik bezeichnet. Ein solcher Unterricht will jungen Menschen helfen, „religiöse Orientierung“ in einem heterogenen Umfeld zu gewinnen. ${ }^{31}$ Oder in den Worten der Autorengruppe eines Modells für Bildungsstandards und Kompetenzen im evangelischen Religionsunterricht:

\begin{abstract}
Der Religionsunterricht richtet sich auf das gesellschaftlich vorfindliche und identifizierbare Phänomen Religion bzw. Religionen sowie auf religiöse Praxis in unterschiedlichen Erscheinungsformen einschließlich der individuellen religiösen Überzeugungen und Ausdrucksgestalten. [...] Es geht im Religionsunterricht um die Reflexion einer Praxis und um den urteilsfähigen Zugang zu dieser Praxis [...] Nicht die Verpflichtung auf einen Glauben ist das Unterrichtsziel, sondern die Befähigung zur Identifizierung und zum situativ angemessenen Gebrauch religiöser Sprache und religiösen Ausdrucks, auch in Analogie zu oder Unterscheidung von anderen Modi des Weltverstehens. ${ }^{32}$
\end{abstract}

In der Praxis können sich diese Varianten durchaus überschneiden. Auch sind, vergleicht man Deutschland und Frankreich, Entwicklungen zu erkennen: in Deutschland in den letzten Jahrzehnten von Variante $2 \mathrm{zu}$ Variante 4, in Frankreich in den jüngsten Diskussionen über Religion als Gegenstandsfeld staatsbürgerlicher Erziehung von Variante $1 \mathrm{zu}$ Variante 3.

Mit Blick auf die oben angesprochenen Erfordernisse religiöser Bildung dürften die Varianten 1 und 2 in einer religiös pluralistischen Gesellschaft im öffentlichen Schulwesen nicht mehr tragfähig sein. Aber auch die auf den ersten Blick bestechende Variante 3 basiert auf äußerst problematischen Annahmen. Alleine schon die Vorstellung, es gebe ein gänzlich neutrales, von den konkreten Religionen völlig unberührtes, dafür aber wissenschaftlich objektiv gesichertes Wissen über Religion, ist eine szientistische Illusion. Überdies wäre aus bildungstheoretischer Perspektive ein Unterricht über Religion, der sich als bloße ,Kunde' versteht und dafür alle identitätsrelevanten Fragen, auf die es keine sicheren Antworten gibt, ausklammern muss, absolut untragbar. Wie sollten sich junge Menschen religiös bilden können, wenn alle dafür wichtigen Fragen nicht Gegenstand des Unterrichts sein können, weil sie umstritten sind?

Letztlich wird für einen schulischen Religionsunterricht, der die Herausforderungen durch religiöse Vielfalt auf bildungsförderliche Weise aufnimmt, nur eine Weiterentwicklung von Variante 4 in Betracht kommen können. In Deutschland ist diese dialogische, auf Perspektivenvielfalt setzende Konzeptualisierung religiöser Bildung in der evangelischen und der katholischen Religionspädagogik zwar stark verankert. Institutionell aber ist die Lage des Religionsunterrichts angesichts der heutigen religiösen Pluralität unbefriedigend.

30. Vgl. Stefan Heil, „Korrelation“, in WiReLex (Wissenschaftlich-Religionspädagogisches Lexikon im Internet), Januar 2015, [https://www.bibelwissenschaft.de/stichwort/100015/] (Stand: 11.3.2017).

31. Vgl. EKD, Religiöse Orientierung gewinnen. Evangelischer Religionsunterricht als Beitrag zu einer pluralitätsfähigen Schule. Eine Denkschrift des Rates der Evangelischen Kirche in Deutschland, Gütersloh, Gütersloh Verlagshaus, 2014.

32. Dietlind Fischer, Volker Elsenbast (Redaktion), Grundlegende Kompetenzen religiöser Bildung. Zur Entwicklung des evangelischen Religionsunterrichts durch Bildungsstandards für den Abschluss der Sekundarstufe I, Münster, Comenius-Institut, 2006, S. 14. 
Seine oben kurz skizzierte rechtliche Stellung hat ihre historischen Wurzeln in einer Zeit, in der es in Deutschland überwiegend religiös homogene Gebiete gab, im Wesentlichen evangelische und katholische, und entsprechend homogene Schülerschaften. Das hat sich schon durch die konfessionelle Durchmischung infolge der Fluchtbewegungen nach dem Zweiten Weltkrieg geändert und noch mehr durch die muslimische Einwanderung. Heute führt diese rechtliche und institutionelle Situation in der Praxis dazu, dass eine normale Schulklasse sich für ,Religion“ in evangelische, katholische, künftig wohl auch muslimische, möglicherweise zudem in großen Städten noch jüdische, christlich-orthodoxe oder buddhistische Lerngruppen aufteilt, ergänzt um diejenigen aus sich als nicht-religiös verstehenden Elternhäusern, die für ein Ersatzfach wie Ethik optieren. Es liegt auf der Hand, dass dies unter schulorganisatorischen Aspekten, aber auch unter dem Gesichtspunkt des interreligiösen Lernens, eine suboptimale institutionelle Form darstellt.

Es wird für die Zukunft des Religionsunterrichts daher von einiger Bedeutung sein, neue Formen der Kooperation, möglicherweise auch der partiellen Integration dieser diversen ,Religionsunterrichte“ zu entwickeln. Mit Blick auf die Lehrerbildung für den Religionsunterricht ist in diesem Zusammenhang der kürzlich zur Diskussion gestellte Vorschlag, an der Humboldt-Universität in Berlin erstmals eine interreligiöse „Fakultät der Theologien“ zu gründen, von einigem Interesse. ${ }^{33}$

Ferner wird zu fragen sein, in welcher Weise über den Religionsunterricht hinaus weitere Fächer einen Beitrag zur religiösen Bildung leisten können. Der Historiker und Geschichtsdidaktiker Frank-Michael Kuhlemann hat in diesem Sinn eine systematische Wiedererschließung der Religionsgeschichte im Geschichtsunterricht und eine entsprechende Neugestaltung der Lehrpläne für das Fach in Deutschland gefordert. Es sei, so Kuhlemann,

erschreckend, wie wenig Schüler und Studenten heute in der Lage sind, grundlegende religionsgeschichtliche Sachverhalte zu benennen. Ob es die Kenntnis des Dekalogs oder die Abfolge zentraler Perioden der Kirchen- und Religionsgeschichte sind, empirische Studien verweisen auf weitreichende Unsicherheit, wenn nicht Unkenntnis. ${ }^{34}$

Es ist zu befürchten, dass vergleichbare Bilanzen des Kunst-, Musik- oder Deutschunterrichts kein wesentlich besseres Bild zeigen würden.

Für hoch religiöse Einwanderer wird es keine überzeugende Integrationsperspektive sein, wenn der aufnehmenden Gesellschaft auf die Gretchenfrage in Goethes Faust - „Nun sag, wie hast du’s mit der Religion?“ keine andere Antwort mehr einfällt als der Hinweis, dies sei Privatsache. Das ist umso weniger attraktiv, wenn schnell zu spüren ist, dass hinter diesem Hinweis in sehr vielen Fällen eine kaum reflektierte religiöse Indifferenz, ja Ahnungslosigkeit in religiösen Fragen steht.

33. Vgl. Rolf Schieder, „Lehren statt bekehren!“, DIE ZEIT, Nr.10 vom 2.3.2017, Beilage „Christ und Welt“, S. 5.

34. Frank-Michael Kuhlemann, „Ohne Religionsgeschichte wird es nicht gehen“, Frankfurter Allgemeine Zeitung, vom 31.1.2015, S. 7. 
Not tut hingegen eine Selbstvergewisserung mit Blick auf die religiösen Grundlagen von Bildung, Schule und freiheitlicher Gesellschaft. In den Worten von Friedrich Wilhelm Graf:

Es geht [...] darum, die Bestände an religiös-moralischer Tradition, die uns (noch) zur Verfügung stehen, reflektiert so präsent zu halten, dass sie der Stärkung einer Kultur der Toleranz dienen..$^{35}$

35. Graf, Götter global, S. 245. 